\title{
Distribuição espacial, sazonal e estrutura populacional do caranguejo Aratus pisonii (H. Milne Edwards) (Crustacea, Decapoda, Sesarmidae) do manguezal de Itacuruçá, Rio de Janeiro, Brasil
}

\author{
Cristiane F. Nicolau ${ }^{1} \&$ Lídia M. Y. Oshiro \\ ${ }^{1}$ Curso de Pós-Graduação em Biologia Animal, Universidade Federal Rural do Rio de Janeiro. E-mail: cris.nicolau@uol.com.br \\ 2 Estação de Biologia Marinha, Universidade Federal Rural do Rio de Janeiro. Rua Sereder, s/ no., Itacuruçá, 23880-000. Rio \\ de Janeiro, Brasil. E-mail: oshiro@ufrrj.br
}

\begin{abstract}
Spatial and seasonal distribution and population structure of the crab Aratus pisonii $(\mathrm{H}$. Milne Edwards) (Crustacea, Decapoda, Sesarmidae) in Itacuruçá mangrove, Rio de Janeiro, Brazil. This work was performed by monthly sampling from June 2000 to May 2001 for population structure studies and by seasonal sampling from January to October 2002 for space and seasonal distribution studies, dividing the mangrove in three different areas. On the space-seasonal distribution study, 694 crabs were caught, where $45 \%$ was captured in area $1,36 \%$ in area 2 and $19 \%$ in area 3 . There were significant differences between the number of individuals between area 2 and 3. For the population structure study a total of 742 crabs were captured, being $59 \%$ male, $32.7 \%$ female and $8.3 \%$ ovigerous females. The sex ratio was 1:0.69 (male:female), and the carapace width ranged from 9.6 to $25.0 \mathrm{~mm} \mathrm{CW}$ in males and from 5.5 to $24.9 \mathrm{~mm} \mathrm{CW}$ in females. The size class distribution was unimodal and the males were more frequent between 19.1 to $21.0 \mathrm{~mm} \mathrm{CW}$, while females between 17.1 to 19 $\mathrm{mm} \mathrm{CW}$. The sex ratio along each size class showed the male predominance in almost all classes except between the classes of 5.1 to 7.0 and 7.1 to $9.0 \mathrm{~mm} \mathrm{CW}$.
\end{abstract}

KEY WORDS. Populational biology; Sepetiba Bay; sex-ratio; tree crab.

RESUMO. Este trabalho teve por objetivo analisar a distribuição espacial e sazonal e a estrutura populacional do caranguejo arborícula Aratus pisonii (H. Milne Edwards, 1837). $\mathrm{O}$ trabalho foi realizado através de coletas mensais de junho de 2000 a maio de 2001, para estudo da estrutura populacional e coletas sazonais de janeiro a outubro de 2002 para o estudo da distribuição espacial e sazonal, dividiu-se o manguezal em três áreas. Para a distribuição espacial e sazonal foram capturados 694 indivíduos sendo $45 \%$ na área 1, 36\% na área 2 e $19 \%$ na área 3. O número de indivíduos apresentou diferença significativa na distribuição espacial entre as áreas 2 e 3. Para o estudo da estrutura populacional foi capturado um total de 742 caranguejos, sendo 59\% machos, 32,7\% fêmeas e 8,3\% fêmeas ovígeras. A razãosexual obtida foi de 1: 0,69 (macho:fêmea) e a largura da carapaça variou de 9,6 a $25,0 \mathrm{~mm}$ em machos e de 5,5 a $24,9 \mathrm{~mm}$ em fêmeas. A distribuição da frequiência em classes de tamanho foi unimodal e os machos foram mais freqüentes entre as classes de 19,1 a $21,0 \mathrm{~mm}$ LC, enquanto as fêmeas entre 17,1 a 19,0 mm LC. A razão sexual ao longo de cada classe de tamanho demonstrou predominância de machos em quase todas as classes, exceto entre as classes de 5,1 a 7,0 e 7,1 a 9,0 mm de LC.

PALAVRAS-CHAVE. Baía de Sepetiba; biologia populacional; caranguejo arborícola; razão sexual.

Os manguezais são ecossistemas de transição entre ambientes terrestres e marinhos típicos de regiões tropicais e subtropicais, sujeitos ao regime das marés e que ocorrem em zonas costeiras (SCHAEFfer - Novelli 1995). Segundo esse mesmo autor, os crustáceos decápodos apresentam uma fauna característica desse ecossistema, possuindo papel importante na cadeia alimentar, decomposição de matéria orgânica e bioturbação do solo.

Nos manguezais da Baía de Sepetiba foi observado um total de cinco espécies de Grapsoidea, sendo uma da família
Grapsidae - Goniopsis cruentata (Latreille, 1803) - e outras quatro da família Sesarmidae: Aratus pisonii (H. Milne Edwards, 1837); Sesarma rectum Randall, 1840; Chasmagnathus granulata Dana, 1851; e Armases rubripes (Rathbun, 1897) (= Metasesarma rubripes) (Oshiro et al. 1998).

Aratus pisonii é um caranguejo que apresenta hábito arborícola, sendo encontrado em ramos e troncos das árvores de manguezal (WARNER 1967). É uma espécie muito abundante, habitando principalmente a borda e o meio do manguezal

Revista Brasileira de Zoologia 24 (2): 463-469, junho 2007 
(Oshiro et al. 1998), sendo conhecido popularmente como marinheiro. Apresenta ampla distribuição geográfica, ocorrendo no Atlântico Ocidental desde a Flórida, Golfo do México, Antilhas, norte da América do Sul, Guianas, até o Brasil (Piauí ao Paraná); e no Pacífico Oriental da Nicarágua até o Peru (Coelho \& Ramos 1972, Melo 1996).

Segundo Beever et al. (1979), A. pisonii é um importante membro da comunidade do mangue vermelho, destruindo e prejudicando as folhas, em função de seu hábito herbívoro. Porém, de acordo com Wilson (1981), Lacerda (1981), Diaz \& Conde (1988), Thomas et al. (1988) e LACERDa et al. (1991), essa espécie também se alimenta de pequenos insetos e, quando possível, de pedaços de animais que podem ser encontrados presos entre as raízes do mangue.

No Brasil as informações acerca dessa espécie têm sido oriundas do Estado de São Paulo, em relação à fecundidade, modos de reprodução, análise comparativa de sua biologia populacional com $S$. rectum e investimento reprodutivo em desovas consecutivas por Leme \& Negreiros-Fransozo (1998a, b) e Leme $(2002,2006)$. No estado do Rio de Janeiro, destacam-se os trabalhos realizados por LACERDA (1981) e LACERDA et al. (1991), relacionados à dieta natural e o de Nicolau \& Oshiro (2002), sobre aspectos reprodutivos.

Este trabalho traz informações sobre a distribuição espacial e sazonal, bem como a estrutura populacional do caranguejo A. pisonii, no Manguezal de Itacuruçá, Baía de Sepetiba, Mangaratiba, Rio de Janeiro, visando contribuir para a ampliação dos conhecimentos sobre esta espécie.

\section{MATERIAL E MÉTODOS}

As coletas foram realizadas no manguezal de Itacuruçá entre as coordenadas $22^{\circ} 54^{\prime}-23^{\circ} 04^{\prime} \mathrm{S}$ e $43^{\circ} 53^{\prime}-44^{\circ} 10^{\prime} \mathrm{W}$, totalizando, aproximadamente, 10 ha de extensão.

As amostragens foram efetuadas em duas etapas, sendo a primeira realizada mensalmente de junho de 2000 a maio de 2001, para o estudo da estrutura populacional, e uma segunda resultante de coletas sazonais em 2002 (outubro/primavera, janeiro/verão, maio/outono e agosto/inverno), sendo nestas o manguezal dividido em três áreas a partir da borda do mangue: 0 m (área 1), 45 m (área 2) e 90 m (área 3). Todas as coletas foram efetuadas durante as marés baixas de sizígia, de acordo com a tábua de marés do Porto de Itaguaí (DHN 2000, 2001, 2002).

As coletas foram efetuadas manualmente utilizando-se o esforço de captura de três coletores durante 50 minutos, nas áreas de 0 a $45 \mathrm{~m}$ para as coletas mensais, e durante $30 \mathrm{minu}-$ tos/área para as coletas sazonais.

Os caranguejos coletados foram devidamente etiquetados e mantidos sob congelamento no laboratório da Estação de Biologia Marinha (EBM/UFRRJ) até o processamento. Após o descongelamento, os caranguejos foram identificados quanto ao sexo, tiveram sua maior largura cefalotorácica (LC) medida com paquímetro, e então depositados na coleção científica de crustáceos da referida Estação de Biologia Marinha.

Revista Brasileira de Zoologia 24 (2): 463-469, junho 2007
A comparação do número de indivíduos/área, bem como de cada sexo/área foram realizadas pelo teste de qui-quadrado $\left(\chi^{2}\right)$, sob 5\% de significância.

A comparação das médias de tamanho de cada sexo por área foi realizada com o teste " $\mathrm{t}$ " de Student, enquanto para confrontar o número de indivíduos entre as estações do ano e as áreas estudadas foi usada uma análise de variância (ANOVA), também a 5\% de significância.

A estrutura populacional foi analisada distribuindo-se os animais em 10 classes de tamanho, em intervalos de $2 \mathrm{~mm}$, numa amplitude de 5 a $25 \mathrm{~mm}$ de LC. Posteriormente, foi verificada a normalidade dos dados pelo teste de KolmogorovSmirnov, a 1\% de significância (ZAR 1984).

A razão sexual populacional foi estabelecida pelo número total de machos em relação ao das fêmeas, além da razão sexual por mês e classes de tamanho. A possível diferença entre os sexos foi verificada pelo $\chi^{2}$, a $5 \%$ de significância.

\section{RESULTADOS}

\section{Estrutura populacional}

Durante um ano foram capturados 742 exemplares de $A$. pisonii, sendo 59\% machos ( $\mathrm{n}=437), 32,7 \%$ fêmeas não ovígeras $(\mathrm{n}=243)$ e $8,3 \%$ fêmeas ovígeras $(\mathrm{n}=62)$ (Tab. I).

Tabela I. Freqüência de machos e fêmeas de $A$. pisonii capturados de junho de 2000 a maio de 2001, a razão sexual (macho:fêmea) e o teste de qui-quadrado (X2), no Manguezal de Itacuruçá, Rio de Janeiro.

\begin{tabular}{|c|c|c|c|c|}
\hline Meses & Machos & Fêmeas & Macho/Fêmea & $\chi^{2}$ \\
\hline Jun/2000 & 22 & $13(0)^{*}$ & $1: 0,6$ & 2,32 \\
\hline Jul & 44 & $25(0)$ & $1: 0,6$ & 2,61 \\
\hline Ago & 26 & $21(1)$ & $1: 0,8$ & 0,16 \\
\hline Set & 26 & $22(9)$ & $0,8: 1$ & 0,21 \\
\hline Out & 25 & $6(6)$ & $1: 0,5$ & 2,28 \\
\hline Nov & 36 & $31(7)$ & $0,9: 1$ & 0,02 \\
\hline Dez & 33 & $8(4)$ & $1: 0,4$ & 4,90 ** \\
\hline $\operatorname{Jan} / 2001$ & 26 & $18(6)$ & $1: 0,9$ & 0,04 \\
\hline Fev & 41 & $12(12)$ & $1: 0,6$ & 2,22 \\
\hline Mar & 66 & $21(16)$ & $1: 0,6$ & $4,08^{* *}$ \\
\hline Abr & 46 & $34(1)$ & $1: 0,8$ & 0,74 \\
\hline Mai & 46 & $32(0)$ & $1: 0,7$ & 1,25 \\
\hline Total & 437 & $243(62)$ & $1: 0,69$ & 23,48 \\
\hline
\end{tabular}

* Número de fêmeas ovígeras entre parênteses; ${ }^{* *} p<0,05$.

O tamanho (LC) dos machos variou de 9,6 a 25,0 mm, com tamanho médio de $18,8 \pm 2,9 \mathrm{~mm}$ enquanto nas fêmeas não ovígeras, essa variação foi de 5,5 a $24,9 \mathrm{~mm}$ e média de $17,3 \pm 2,9 \mathrm{~mm}$. As fêmeas ovígeras apresentaram LC variando de 14,0 a 23,6 $\mathrm{mm}$, com tamanho médio de $19,6 \pm 2,1 \mathrm{~mm}$. 
A distribuição de freqüência dos indivíduos em classes de tamanho (LC) apresentou distribuição normal, segundo o teste de Kolmogorov-Smirnov ( $p>0,01$ ), sendo unimodal.

Os machos foram mais freqüentes entre 19,1 a 21,0 mm, enquanto a maior freqüência para as fêmeas ocorreu entre 17,1 a 19,0 mm (Fig.1).

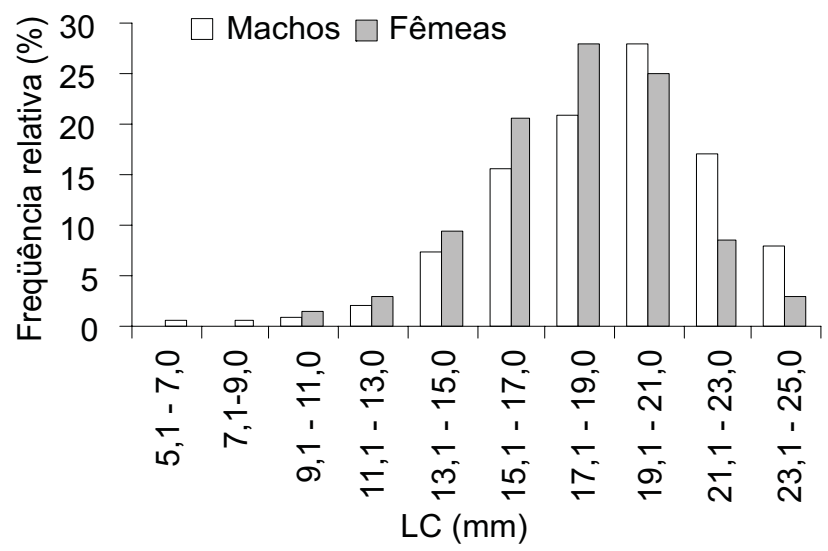

Figura 1. Distribuição da freqüência relativa de machos e fêmeas em classes de tamanhos de $A$. pisonii capturados no período de junho de 2000 a maio de 2001 no Manguezal de Itacuruçá.

O teste " $\mathrm{t}$ " demonstrou que houve diferença significativa no tamanho médio entre os sexos a 5\% de significância. O teste " $\mathrm{t}$ " para duas amostras, revelou variâncias com valores de 8,96 para machos e 9,00 para fêmeas, demonstrando que nos machos ocorreu menor variação do que nas fêmeas.

A razão sexual para a população estudada foi de 1:0,69, com predomínio de machos em relação às fêmeas $\left(\chi^{2}=23,48\right.$; $\mathrm{p}<0,05$ ). Com exceção de setembro e novembro de 2000, os machos foram mais abundantes do que as fêmeas, embora diferenças significativas ( $p<0,05)$ tenham sido verificadas apenas nos meses de dezembro de 2000 e março de 2001 (Tab. I).

Houve predominância de machos em quase todas as classes de tamanho (LC), exceto nas classes de 5,1 a 7,0 e de 7,1 a 9,0 $\mathrm{mm}$ de LC (Tab. II).

Na tabela III é apresentada a razão sexual obtida por diferentes autores para essa espécie, onde foi comum o desvio da razão sexual em favor das fêmeas, discordando do presente estudo.

\section{Distribuição espacial e sazonal}

Foi capturado um total de 694 indivíduos de A. pisonii, sendo 363 machos, 242 fêmeas não ovígeras e 89 fêmeas ovígeras. A distribuição foi diferenciada entre as áreas, com freqüência de $45 \%$ na área $1,36 \%$ na área 2 e $19 \%$ na área $3 . \mathrm{Na}$ área 1 , mais próxima à franja do mangue $(0-45 \mathrm{~m})$ foi verificada maior freqüência da espécie, independente do sexo (Fig. 2).

Comparando-se a população total, o número de machos e de fêmeas entre as áreas 1, 2 e 3 pode -se observar pelo teste

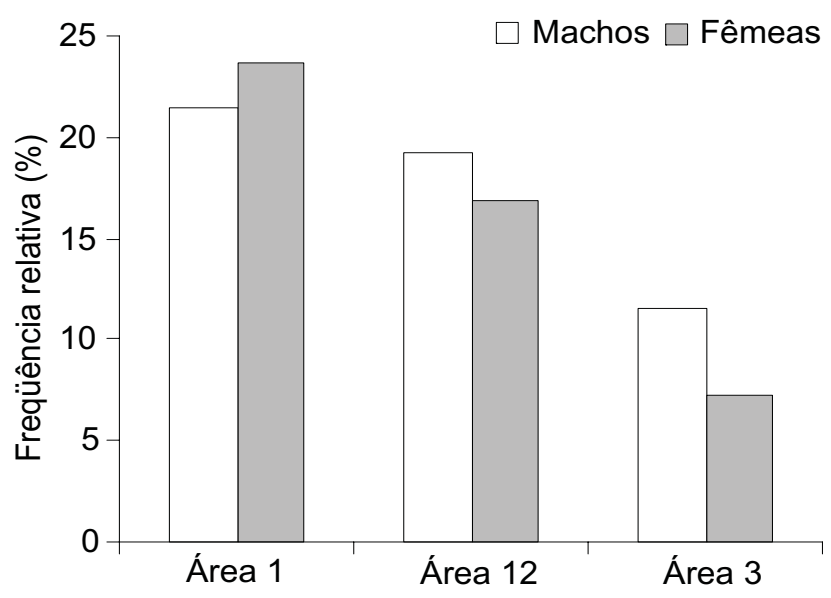

Figura 2. Distribuição da freqüência relativa de machos e fêmeas nas áreas 1, 2 e 3, de $A$. pisonii coletados no período de janeiro a outubro de 2002 no Manguezal de Itacuruçá, Rio de Janeiro.

Tabela II. Freqüência de machos e fêmeas e razão sexual de acordo com as classes de largura da carapaça de $A$. pisonii, capturados de junho de 2000 a maio de 2001, no manguezal de Itacuruçá, Rio de Janeiro.

\begin{tabular}{cccc}
\hline Largura da Carapaça $(\mathrm{mm})$ & Machos & Fêmea & Macho/fêmea \\
\hline $5,1-7,0$ & 0 & $2(0)^{*}$ & $0: 2,0$ \\
$7,1-9,0$ & 2 & $4(0)$ & $1: 2,0$ \\
$9,1-11,0$ & 12 & $8(0)$ & $1: 0,7$ \\
$11,1-13,0$ & 13 & $10(0)$ & $1: 0,8$ \\
$13,1-15,0$ & 30 & $27(1)$ & $1: 0,9$ \\
$15,0-17,0$ & 78 & $54(9)$ & $1: 0,8$ \\
$17,1-19,0$ & 91 & $68(18)$ & $1: 0,9$ \\
$19,1-21,0$ & 114 & $55(21)$ & $1: 0,7$ \\
$21,1-23,0$ & 72 & $11(10)$ & $1: 0,3$ \\
$23,1-25,0$ & 25 & $4(3)$ & $1: 0,3$ \\
\hline
\end{tabular}

* Número de fêmeas ovígeras, entre parênteses.

Tabela III. Razão sexual de A. pisonii em diferentes manguezais de áreas geográficas distintas.

\begin{tabular}{clc}
\hline \multicolumn{1}{c}{ Autor } & \multicolumn{1}{c}{ Local } & $\begin{array}{c}\text { Razão sexual } \\
\text { (macho:fêmea) }\end{array}$ \\
\hline CONDE \& DíAz (1989) & $\begin{array}{l}\text { Laguna de Tacarigua } \\
\text { National Park } \\
\text { (Venezuela) }\end{array}$ & $1: 1,6$ \\
DíAz \& Conde (1989) & $\begin{array}{l}\text { Morrocoy National Park } \\
\text { (Venezuela) }\end{array}$ & $1: 1,3$ \\
Leme et al. (2002) & $\begin{array}{l}\text { Ubatuba (Brasil) } \\
\text { Presente estudo }\end{array}$ & $1: 1,2$ \\
\hline
\end{tabular}

de qui-quadrado que apenas machos das áreas 1 e 2 não apresentavam diferença significativa $(p>0,05)$ (Tab. IV).

$\mathrm{Na}$ área 1 houve predomínio de indivíduos entre 19,1 a 21,0 $\mathrm{mm}$, independente do sexo, similarmente ao verificado

Revista Brasileira de Zoologia 24 (2): 463-469, junho 2007 
Tabela IV. Número de indivíduos de cada sexo de A. pisonii por área e comparação entre o múmero de indivíduos entre áreas pelo teste de qui-quadrado, capturados de janeiro a outubro de 2002, no Manguezal de Itacuruçá, Rio de Janeiro.

\begin{tabular}{|c|c|c|c|c|c|}
\hline & Áreas & $\begin{array}{l}\text { No de indivíduos em cada } \\
\text { área }\end{array}$ & Áreas comparadas & $\begin{array}{l}\text { Comparação entre } \mathrm{n}^{\circ} \text { de indivíduos } \\
\text { capturados em cada área }\end{array}$ & $\chi^{2}$ \\
\hline \multirow[t]{3}{*}{ População total } & 1 & 313 & $1 \times 2$ & $313 \times 251$ & * \\
\hline & 2 & 251 & $1 \times 3$ & $313 \times 130$ & * \\
\hline & 3 & 130 & $2 \times 3$ & $251 \times 130$ & * \\
\hline \multirow[t]{3}{*}{$N^{\circ}$ de machos } & 1 & 149 & $1 \times 2$ & $149 \times 134$ & ns \\
\hline & 2 & 134 & $1 \times 3$ & $149 \times 80$ & * \\
\hline & 3 & 80 & $2 \times 3$ & $134 \times 80$ & * \\
\hline \multirow[t]{3}{*}{$N^{o}$ de fêmeas } & 1 & 164 & $1 \times 2$ & $164 \times 117$ & * \\
\hline & 2 & 117 & $1 \times 3$ & $164 \times 50$ & * \\
\hline & 3 & 50 & $2 \times 3$ & $117 \times 50$ & * \\
\hline
\end{tabular}

*) $p<0,05 ; n s) p>0,05$.

nas áreas 2 e 3, onde predominou a classe de 17,1 a 19,0 mm (Fig. 3). Não foram verificadas diferenças significativas quando comparadas as médias de tamanho entre os sexos de cada área $(\mathrm{p}>0,05)$.

A freqüência relativa dos machos foi superior na área 1, em todas as estações do ano, exceto na primavera, quando a maior freqüência ocorreu na área 2 . No entanto, as fêmeas foram mais freqüentes na área 1 em todas as estações do ano (Fig. 4). Não foi evidenciada diferença significativa para o número total de indivíduos entre as estações do ano e áreas estudadas.

\section{DISCUSSÃO}

Um dos aspectos mais importantes na caracterização da estrutura de população de uma espécie é a distribuição de seus exemplares em classes de tamanho, e as proporções de seus membros ao longo de cada classe (PIANKa 1974).

Em Ubatuba (São Paulo), Leme (2002) observou que a população de $A$. pisonii apresentou indivíduos de menor porte, iniciando com cerca de $4 \mathrm{~mm}$, sendo pouco menor do que aqueles do presente estudo, enquanto as fêmeas ovígeras ocorreram com tamanho muito similar. Para Diaz \& Conde (1989), na Venezuela, as fêmeas ovígeras também apresentaram variação de tamanho similar (11,0 a 24,0 mm), mostrando que a estrutura populacional dessa espécie pode variar pouco conforme a localização geográfica ou condição estrutural dos manguezais.

A diferença no tamanho mínimo dos animais encontrados no presente estudo, pode ter sido decorrente de erro amostral dos exemplares de menor porte, fato devido, certamente, à coloração dos jovens ser muito semelhante à do substrato, além de serem mais ágeis, dificultando a captura. Este aspecto foi anteriormente ressaltado por HARTNOLL \& BRYANT (1990), onde a ausência ou reduzido número de indivíduos nas menores classes de tamanho, tem sido comum em estudos de estrutura populacional dos decápodos.

Segundo Warner (1967) e Diaz \& Conde (1989), a distribuição de freqüência de tamanho unimodal, tanto para ma-
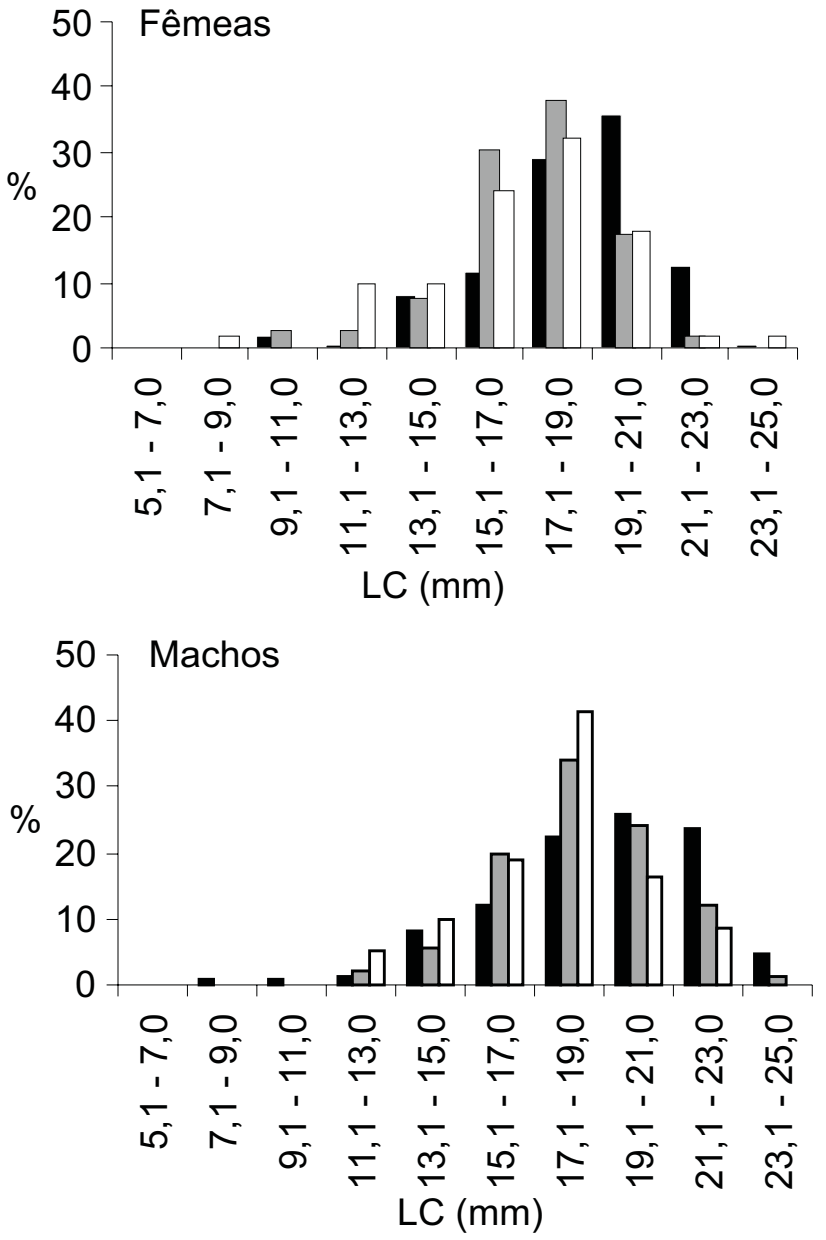

Área $1 \square$ Área $2 \square$ Área 3

Figura 3. Distribuição da freqüência relativa em classes de tamanho de machos e fêmeas de $A$. pisonii nas áreas 1, 2 e 3 coletados no período de janeiro a outubro de 2002 no Manguezal de Itacuruçá. 

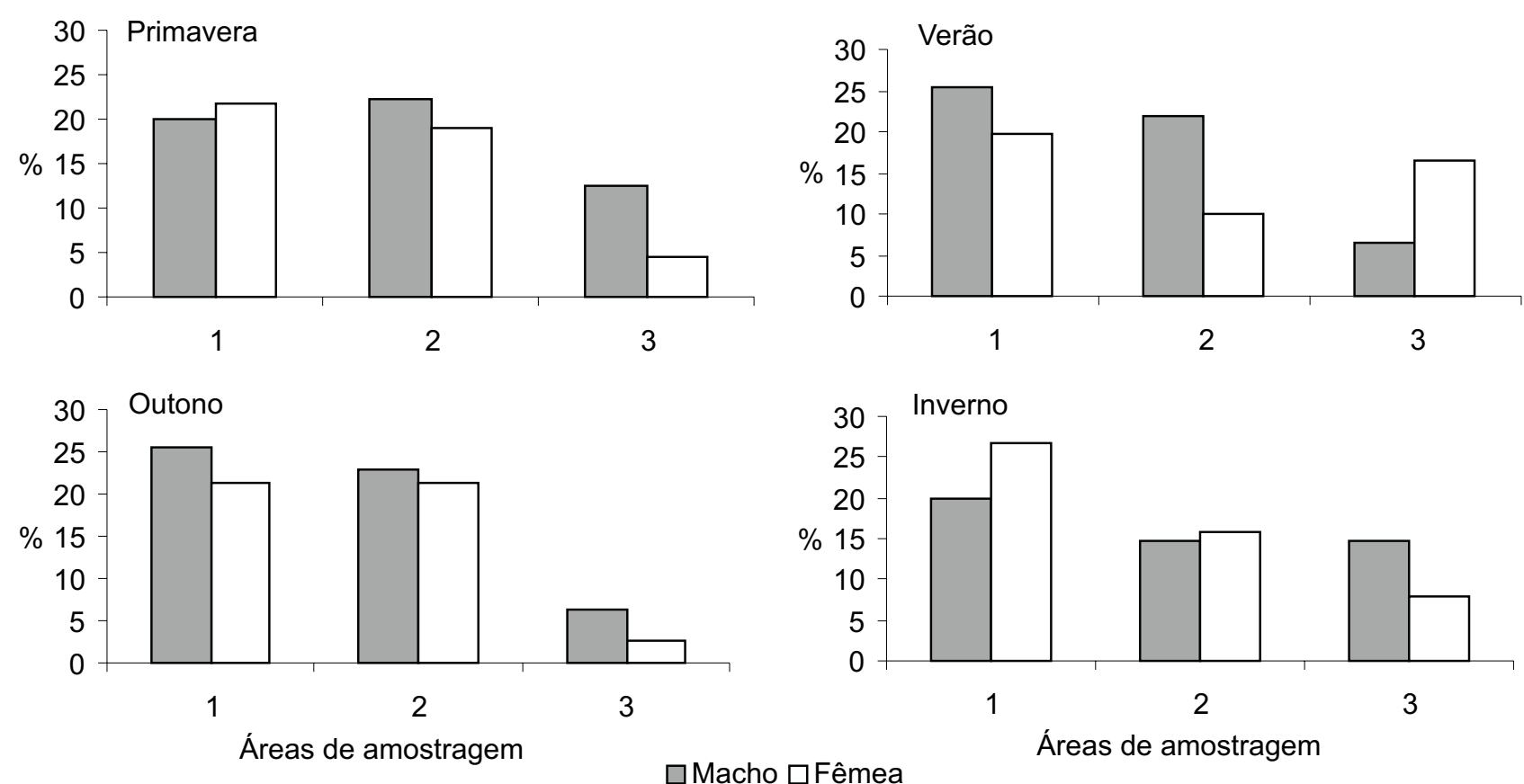

Figura 4. Distribuição da freqüência relativa de machos e fêmeas de A. pisonii nas áreas 1, 2 e 3 de acordo com as estações do ano, coletados no período de janeiro a outubro de 2002 no Manguezal de Itacuruçá.

chos como para fêmeas, é também comum em decápodos de regiões tropicais, onde as variações climáticas são pouco acentuadas. A distribuição unimodal, normalmente reflete um recrutamento contínuo, sem rompimentos de classes e taxas de mortalidade constantes (Díaz \& Conde 1989).

$\mathrm{O}$ fato de se encontrar um maior número de machos em relação às fêmeas nas duas maiores classes de tamanho, pode estar relacionado com o crescimento mais lento das fêmeas em relação aos machos, após a puberdade (WARNER 1967, WENNER 1972, Thurmam 1985, Fukui 1988, Conde \& Diaz 1989b, Diaz \& Conde 1989). Ao incubarem seus ovos, as fêmeas estão privadas de seu crescimento somático, como resultado do antagonismo entre a reprodução e crescimento (ADIYODI \& ADIYoDI 1970), com potencialização do período de intermuda, explicando o maior número de fêmeas nas principais classes reprodutivas (WARNER 1967, Diaz \& Conde 1989, Conde \& Diaz 1989a).

A proporção entre os sexos pode diferir em relação a vários fatores, podendo afetar mais um sexo do que outro (Wilson \& PiAnKa 1963, Wenner 1972).

Segundo Góes \& Fransozo (2000), a equivalência na proporção de machos e fêmeas, pode variar muito segundo a família de Brachyura. Tais autores afirmam, também, que a taxa de crescimento, a pressão ambiental, a disponibilidade de alimento e a estratégia reprodutiva, podem afetar os sexos em diferentes extensões, promovendo, assim, proporções diferenciadas entre os sexos ao longo de sua ontogenia.

De acordo com Wilson \& Pianka (1963), Wenner (1972) e
Haley (1979), diferentes taxas de crescimento e mortalidade promovem desvios na razão sexual ao longo do tempo; e da mesma forma, os períodos de restrição alimentar para um dos sexos, também pode contribuir para o desequilíbrio das taxas de mortalidade e provocar desvios da razão sexual. Segundo esses mesmos autores, mecanismos de migração associados à alimentação, defesa ou reprodução também podem pressionar a ocupação de micro-habitats distintos entre os sexos, atuando sincronicamente para desvios na razão sexual, em uma população.

Segundo Diaz \& Conde (1989), o efeito da predação e sua influência sobre as taxas de mortalidade natural é muito difícil de ser avaliado, pois a predação por pássaros ou outros caranguejos (e.g. G. cruentata, segundo WARNER 1977) parece não ser uma causa importante à mortalidade de $A$. pisonii, ao contrário da predação por peixes devido a fuga destes para a água.

Em relação à distribuição espacial, o presente estudo corrobora OsHiro et al. (1998), que mencionaram a maior presença de A. pisonii nas franjas de manguezal da Baía de Sepetiba, sempre sobre as árvores. Segundo Coelho (1963) e Branco (1991), essa espécie é frequentemente encontrada em ramos e troncos no mesolitoral, o que também corroborando o presente estudo.

Variações de salinidade, da temperatura do ar/água e da composição/textura do sedimento, também podem limitar a distribuição de espécies no ambiente (ABELE 1974, Fransozo et al. 1992).

De acordo com Frusher et al. (1994), caranguejos sesarmídeos têm padrões de distribuição distintos nos manguezais, sendo que a tolerância à salinidade e habilidade de osmoregula- 
ção não refletem adequadamente sua distribuição nos manguezais, enquanto fatores como a competição intraespecífica e predação, provavelmente influenciam a abundância da espécie no manguezal.

Segundo Díaz \& Conde (1989) quando as larvas alcançam a fase adulta, o caranguejo adquire independência da salinidade da água, embora fique à mercê do ambiente de manguezal no que tange à sua reprodução.

Conforme o trabalho realizado por Oshiro et al. (1998), nos manguezais da Baía de Sepetiba, verificou-se que a Superfamília Grapsoidae encontra-se distribuída basicamente entre a borda e o meio do manguezal, evidenciando uma nítida preferência ecológica para cada espécie.

Wilson (1989) estudou a distribuição de quatro espécies de caranguejos de manguezal no sul da Flórida, verificando que o caranguejo arborícola $A$. pisonii, ocupava, principalmente, a superfície do sedimento no manguezal e as raízes da vegetação arbórea. Segundo esse mesmo autor, o risco de predação foi muito maior na superfície do sedimento ou em raízes de manguezal, bem como na maré alta do que na baixa, ocorrendo riscos diferenciados de predação dentro deste ecossistema.

Em relação à distribuição sazonal, pode-se concluir que não houve preferência dessa espécie por determinadas áreas do manguezal estudado com a estação do ano, o que pode ser verificado pela presença da espécie durante todo o ano nas três áreas analisadas.

O presente trabalho permitiu concluir que a população de A. pisonii do manguezal de Itacuruçá, apresentou número de machos pouco mais elevado do que de fêmeas, e que o tamanho dos exemplares foram similares ao de outros manguezais já estudados. Em relação à distribuição espacial, a espécie mostrou maior preferência pela franja do que pelo meio do manguezal.

\section{AGRADECIMENTOS}

A Casimiro A. Alves, Estação de Biologia Marinha, UFRRJ, Zilanda de S. Silva, Giovana V. Lima, Willianm C. Rodrigues e Maria C. Ferreira.

\section{REFERÊNCIAS BIBLIOGRÀFICAS}

Abele, G.L. 1974. Species diversity of decapod crustaceans marine habitats. Ecology 55: 156-161.

Adiyodi, K.G. \& R.G. Adiyodi. 1970. Endocrine control of reproduction Decapod Crustacea. Biological Review 45: 121-165.

Beever, J.W. III; D. Simberloff. \& L.L. King. 1979. Herbivory and predation by the mangrove tree crab Aratus pisonii. Oecologia 43: 317-328.

Branco, J. O. 1991. Aspecto Ecológico dos Brachyura (Crustacea, Decapoda) no Manguezal do Itacorubi, Santa Catarina, Brasil. Revista Brasileira de Zoologia 7 (1-2): 165-179.

Coelho, P.A. 1963. Distribuição dos Crustáceos Decapódos na área de Barradas

Jangadas. Trabalhos Oceanográficos da Universidade Fede- ral de Pernambuco 5/6: 159-173. [1964]

Coelho, P.A. \& M.A. Ramos. 1972. A constituição e a distribuição da fauna de decápodos do litoral leste da América do Sul, entre latitudes $5^{\circ} \mathrm{N}$ e $39^{\circ} \mathrm{S}$. Trabalhos Oceanográficos da Universidade Federal de Pernambuco 13: 133-236.

CONDE, J.E. \& H. DiAz. 1989a. The mangrove tree crab Aratus pisonii in a tropical estuarine coastal lagoon. Estuaruarine Costal and Shelf Science 28: 639-650.

Conde, J.E. \& H. Diaz. 1989 b. Productividad del habitat e historias de vida del cangrejo de mangle Aratus pisonii (H. Milne Edwards) (Brachyura: Grapsidae). Boletin del Instituto Oceanográfico de la Universidade Del Oriente 28 (1/2): 113-120.

DhN. 2000. Diretoria de Hidrografia e Navegação. Tábua de marés. Costa do Brasil e Portos Estrangeiros. Rio de Janeiro, Diretoria de Hidrografia e Navegação da Marinha do Brasil, p. 117-119.

DhN. 2001, 2002. Diretoria de Hidrografia e Navegação. Tábua de marés. Costa

do Brasil e Portos Estrangeiros. Rio de Janeiro, Diretoria de Hidrografia e Navegação da Marinha do

Brasil, p. 120-122.

Díaz, H. \& J.E. Conde. 1988. On the foods sources for the mangrove crab Aratus pisonii (Brachyura, Grapsidae). Biotropica 20 (4): 348-350.

Diaz, H. \& J.E. Conde. 1989. Populations dynamics and life history of the mangrove crab Aratus pisonii (Brachyura: Grapsidae) in a marine environment. Bulletin of Marine Science 45 (1): 148-163.

Fransozo, A.; M.L. Negreiros-Fransozo; F.L.M. Mantelatto; M.A.A. Pinheiro. 1992. Composição e distribuição dos brachyuras (Crustacea, Decapoda) do litoral não consolidado na Enseada da Fortaleza, Ubatuba (SP). Revista Brasileira de Biologia 52 (4): 667-675.

FukuI, Y. 1988. Comparative studies on the life history of the grapsid crabs (Crustacea, Brachyura) inhabiting intertidal cobble and bouder shores. Publication of the Seto Marine Biological Laboratory 33 (4/6): 121-162.

Frusher, S.D.; R.I. Giddins.; T.J. SMITH III. 1994. Distribution and abundance of grapsid crabs (Grapsidae) in a mangrove estuary: effects of sediment characteristics, salinity tolerances, and osmoregulatory ability. Estuaries 17 (3): 647-654.

Góes, J.M. \& A. Fransozo. 2000. Sex ratio analysis Eriphia gonagra (Decapoda, Xanthidae). Iheríngia, Série Zoologia 88: 151157.

Haley, S.R. 1979. Sex ration as a fuction of size in Hippa pacifica Dana (Crustacea, Anomura, Hippidae): a test of sex reversal and differential growth rate hypotheses. Americam Naturalist 113 (3): 391-397.

HaRTNOll, R.G. \& A.D. Bryant. 1990. Size-frequency distribuitions in decapod Crustacea. The quick, the dead, and the castoffs. Journal of Crustacean Biology 10 (1): 14-19.

LACERDA, L.D. 1981. Mangrove wood pulp, an alternative food source for the tree-crab Aratus pisonii. Biotropica 13 (4):

Revista Brasileira de Zoologia 24 (2): 463-469, junho 2007 
137.

LACERDA, L.D.; C.A.R. Silva.; C.E. Rezende \& L.A. Martinelli. 1991. Food sources for the mangrove tree crab Aratus pisonii: a carbon isotopic study. Revista Brasileira de Biologia 51 (3): 685-687.

LEME, M.H. DE A. 2002. A Comparative analysis of the population biology of the mangrove crabs Aratus pisonii and Sesarma rectum (Brachyura, Grapsidae) from the north coastal of São Paulo state, Brazil. Journal of Crustacean Biology 22 (3): 553-557.

LEME, M.H. DE A. 2006. Investimento reprodutivo e produção de ovos em desovas consecutivas do caranguejo Aratus pisonii (H. Milne Edwards) (Crustacea, Brachyura, Grapsoidea). Revista Brasileira de Zoologia 23 (3): 727-732.

Leme, M.H. De A. \& M.L. Negreiros-Fransozo. 1998 a. Fecundity of Aratus pisonii (Decapoda: Grapsidae) in the Ubatuba region, State of São Paulo, Brazil., Iheringia Série Zoologia 84: 73-77.

Leme, M.H. de A. \& M.L. Negreiros-Fransozo. 1998 b. Reproductive patterns of Aratus pisonii (Decapoda: Grapsidae) from estuarine area of São Paulo Northern Coast, Brazil. Revista de Biologia Tropical 46 (3): 673-678.

Melo, G.A.S. 1996. Manual de Identificação dos Brachyura (Caranguejos e Siris) do Litoral Brasileiro. São Paulo, Ed. Plêiade, FAPESP, Museu de Zoologia, Universidade de São Paulo, 603p.

NicolaU, C.F. \& L.M.Y. Oshiro. 2002. Aspectos reprodutivos do caranguejo Aratus pisonii (Crustacea, Decapoda, Grapsidae) do manguezal de Itacuruçá, Rio de Janeiro. Revista Brasi- leira de Zoologia 19 (2): 167-173.

Oshiro. L.M.Y.; R. Silva \& Z.S. Silva. 1998. Composição da fauna de braquiúros (Crustaea, Decapoda, Grapsidae) da Baía de Sepetiba - RJ. Nauplius 6: 31-40.

PIANKA, E. R. 1974. Evolutionary ecology. New York, Harper \& Row Publishers, 356p.

SChaeffer-Novelli, Y. 1995. Manguezal - ecossistema entre a terra e o mar. São Paulo, EDUSP, 64p.

Thomas, D.W.; D. Cloutier.; M. Provencher. \& C. Houle. 1988. On the food sources for the mangrove tree crab Aratus pisonii (Brachyura: Grapsidae). Biotropica 20 (4): 348-350.

Thurman, C.L. 1985. Reproductive biology and population structure of the fiddler crab Uca subcylindrica (Stimpson). Biological Bulletin 169: 215-229.

WARnER, G.F. 1967. The life history of the mangrove tree crab Aratus pisonii. Journal of Zoology 153: 321-335.

WARNER, G.F. 1977. The biology of crabs. London, Elek Science, 202p.

Wenner, A.M. 1972. Sex ratio as a function of size in Marine Crustacea. The American Naturalist 106 (4): 321-350.

Wilson, K.A. 1981. Tidal associated feeding in the mangrove tree crab Aratus pisonii. The American Zoologist 21: 105.

Wilson, K.A. 1989. Eology of mangrove crabs: Predation, physical factors and refuges. Bulletin of Marine Science 44: 263-273.

Wilson, M.F. \& E.R. PiAnKa. 1963. Sexual seletion, sex-ratio and mating system. The Americam Naturalist 97: 405-407.

ZAR, J.H. 1984. Bioestatistical analysis. Englewood Cliffs, Prentice-Hall, $2^{\text {nd }}$ ed., 719p.

Recebido em 28.VII.2007; aceito em 03.VI.2007. 